\title{
Cloudy with a chance of peptides: accessibility, scalability and reproducibility with cloud-hosted environments
}

\author{
Benjamin A. Neely ${ }^{1 *}$ \\ ${ }^{1}$ Chemical Sciences Division, National Institute of Standards and Technology, Charleston, SC, \\ USA
}

*Corresponding author: BN; benjamin.neely@nist.gov

\section{Abstract}

Cloud-hosted environments offer known benefits when computational needs outstrip affordable local workstations, enabling high-performance compute without a physical cluster. What has been less apparent, especially to novice users, is the transformative potential for cloud-hosted environments to bridge the digital divide that exists between poorly funded and well-resourced laboratories, and to empower modern research groups with remote personnel and trainees. Using cloud-based proteomic bioinformatic pipelines is not predicated on analyzing thousands of files, but instead can be used to improve accessibility during remote work, extreme weather or working with under-resourced remote trainees. The general benefits of cloud-hosted environments also allow for scalability and encourage reproducibility. Since one possible hurdle to adoption is awareness, this paper is written with the non-expert in mind. The benefits and possibilities of using a cloud-hosted environment are emphasized by describing how to setup an example workflow to analyze a previously published label-free data-dependent acquisition mass spectrometry data set of mammalian urine. Cost and time of analysis are compared using different computational tiers, and important practical considerations are described. Overall, cloud-hosted environments offer the potential to solve large computational problems, but more importantly can enable and accelerate research in smaller research groups with inadequate infrastructure and suboptimal local computational resources.

\section{TOC Graphic}

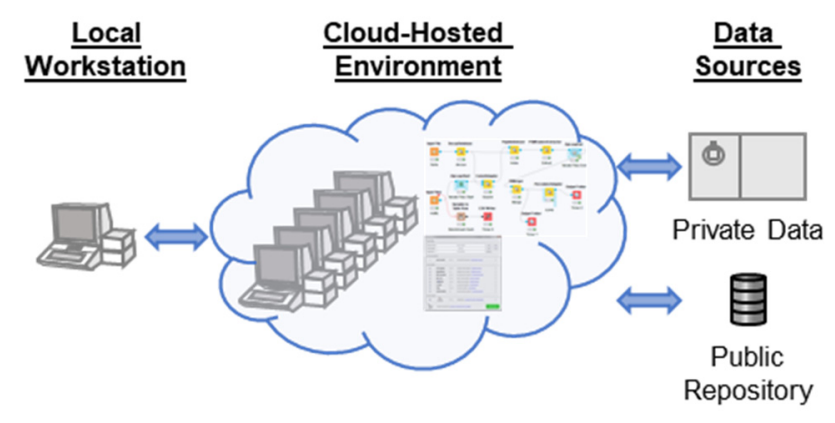

Keywords: cloud computing, remote access, high-performance computing, proteomic workflow 


\section{Introduction}

Remote hosted computational environments, often referred to as the cloud, allow for potentially easier setup, faster processing, and lower cost to build and manage than local workstations. Recently, with increased remote work due to pandemic driven lockdowns, globalization of scientific research (e.g., globally distributed research consortia), and the growth of public data repositories and re-analysis of public data sets, using cloud-hosted environments as the computational backbone of research offers increasing advantages. Specific to proteomics there are opportunities for increased use of cloud-hosted environments, leading to improved accessibility, scalability and reproducibility. Currently, there is a plethora of cloud-based options and different benefits, with real and perceived bottlenecks to implementation. These benefits are not limited to utilizing cloud-hosted environments for high-throughput large-scale proteomics data, but can also prove essential for remote work, remote training or disaster resilience. To help the reader, a glossary of terms is provided.

\section{Cloud Computing}

Depending on the available resources of both investigators and their institution/company, there exists a spectrum of local computational capacity. These resources may be available via remote access, but many cases exist when this is not possible often due to institutional security concerns. By utilizing cloud-hosted environments (Figure 1), computational work may continue even when local resources are inaccessible (due to lockdowns, extreme weather, inconsistent power, etc.) and provide access to remote colleagues and trainees (including those in other countries) that otherwise could be using inadequate local resources. Furthermore, using cloudhosted environments allows users to quickly scale resources to accomplish larger tasks at appropriate times, instead of purchasing or upgrading local hardware that runs far below its potential most of the time and requires periodic system hardware and software maintenance. Depending on the cloud environment, adding computational resources can be as simple as "building" a new virtual cluster with the click of a button, using an instance with more cores or memory with an existing image, utilizing workflows that automatically scale across a virtual cluster depending on workload, or executing a function in a serverless framework. In theory, this means that every researcher can have the same computational capacity, regardless of location.

Cloud-based computing can trace a line from the time-sharing of the 1950 s and $1960 \mathrm{~s}^{1}$ through the packet radio van of $1977^{2}$ into the modern "cloud" available from public and commercial providers. In many regions across the globe, there are federally subsidized resources available to researchers through varied application processes. For instance, Jetstream (a distributed collaboration of the University of Indiana, University of Texas at Austin, and University of Arizona) on the National Science Foundation-funded Extreme Science and Engineering Discovery Environment (XSEDE) allows users to start instances using a catalog of Linux-based images, many already preconfigured for common research applications, and create single multiCPU and high memory instances or multiple-node virtual clusters, and access these by webbased console or remote desktop. Setting up seemingly complicated virtual clusters can be accomplished with ease when a tutorial exists, exemplified by MAKER on Jetstream ${ }^{3}$, Cactus on Amazon Web Services (AWS) ${ }^{4}$, and Bioconductor on AWS ${ }^{5}$. The ease of using a service like 
Jetstream for a cloud novice cannot be understated. Similar to XSEDE, other publicly subsidized resources including ACI-REF (Advanced Cyberinfrastructure Research and Education Facilitators) Network, ELIXIR (the European life-sciences Infrastructure for biological Information), $\mathrm{NCl}$ (National Computational Infrastructure) Australia, and PRACE (Partnership for Advanced Computing in Europe) make free hosted computation time available to researchers. Although the free or low-cost nature of these services is preferred, commercial resources are also available and include AWS, Microsoft Azure and Google Cloud, which also have free tiers or trials available. These services, both public and commercial, are the backbone of most large computational efforts from particle physics to population genomics.

\section{Proteomics in the Cloud}

Mass spectrometry-based proteomics is a broad term encompassing many applications ${ }^{6}$ used across different biological systems ${ }^{7}$, and accordingly has an abundance of software tools available $^{8}$. Protein inference is made possible by peptide identification following database searching of tandem mass spectrometry data ${ }^{9}$. Computation in many modern protein identification algorithms is performed in RAM with high-speed CPUs and varied I/O requirements. These computational requirements mean that high-performance local machines are perceived as better suited to database searching, as opposed to cloud-hosted environments. In contrast, nucleic acid sequencing computation historically relied on massive parallelization on modestly appointed motherboards, and therefore was very amenable to cloud applications. Despite this historic precedent, with decreasing cloud computing costs there is an ever increasing list of cloud-based proteomic solutions including Bolt ${ }^{10}$ and ionbot ${ }^{11}$. More importantly, there are software platforms specifically tuned to run proteomic data processing and analysis in the cloud, such as Galaxy- $\mathrm{P}^{12}$, 13. Even without being specifically tailored to a cloud environment, any software can be used in a cloud-hosted environment, though there may be concerns for licensing. Software developed natively in Linux is best for services like Jetstream or containers like Singularity, while Windows-based applications may be used for services like AWS or containers like Docker. Examples of free or open-source proteomic software that can run in a Linux environment include Crux ${ }^{14}$, EncyclopeDIA ${ }^{15}$, FragPipe ${ }^{16,17}$, ProteoWizard ${ }^{18}$, SearchGUI ${ }^{19}$, The OpenMS Proteomics Pipeline (referred to as OpenMS or TOPP) ${ }^{20,21}$, Trans-Proteomic Pipeline (TPP) ${ }^{22,23}$, and X!Tandem ${ }^{24}$. Other tools that run in Windows but can also be run via command line in a Linux environment include MaxQuant ${ }^{25,26}$, MetaMorpheus ${ }^{27}$, and Spritz ${ }^{28}$. In order to truly take advantage of the scalability of cloud environments, software that can work in a clustered environment is preferred. This relies on distributing tasks across nodes, often integrated with workflow engines including the Konstanz Information Miner (KNIME) ${ }^{29}$, Makeflow ${ }^{30}$, Nextflow ${ }^{31}$, Snakemake ${ }^{32}$, Swift ${ }^{33}$, and Toil ${ }^{34}$, which frequently provide tutorials specific to scaling in cloud-hosted environments. Most proteomic applications have not made the transition to being capable of fully utilizing modern clustering options, though database searching has been shown to benefit greatly from parallelization ${ }^{35}$. Notable non-commercial exceptions include SEQUEST-PVM ${ }^{36}$, UltraQuant, which uses Snakemake to run MaxQuant ${ }^{37}$, and OpenMS-based tools, which can be run on a cluster using $\mathrm{KNIME}^{38}$ and Nextflow ${ }^{39}$. Beyond workflows and virtual clusters, in the coming years computational steps will be offloaded onto serverless frameworks (i.e., function as a service; FaaS $)^{40}$, blurring the line between local and cloud-hosted environments. Given the potential of 
cloud-hosted environments, it seems that we are on the cusp of seeing a shift to cloud-based solutions in proteomics.

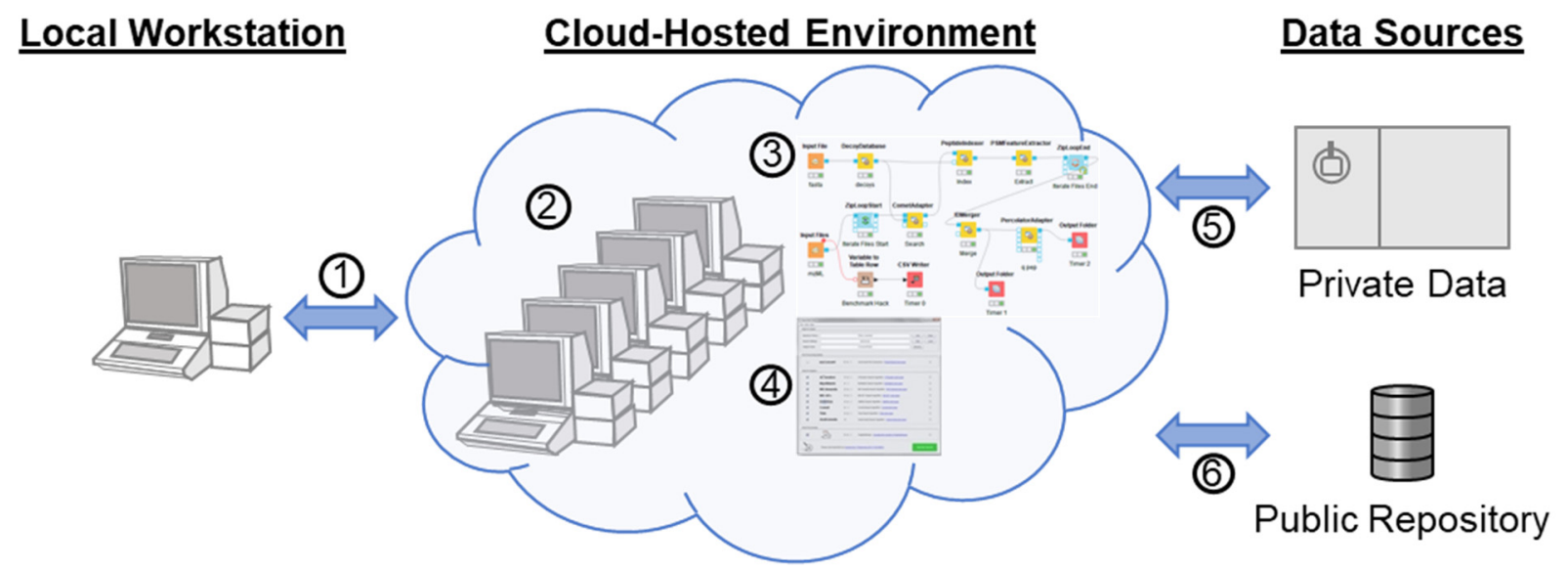

Figure 1. Generalized concept of proteomics in a cloud-hosted environment. The environment is accessed via any client with internet access (1). Data for analysis can be retrieved directly to the environment from private data sources (5) and public data repositories (6). Computational resources can be assigned to the environment prior to analysis (2), at which point programs (4) or workflows (3) may be used to complete the analysis.

\section{Testing Performance in the Cloud}

It has been said that the future is already here, it is just not evenly distributed yet (paraphrased from William Gibson), which is especially true of cloud computing. Research in numerous fields including materials science, astronomy and genomics rely heavily on cloud-based computing, while it is largely absent in proteomic research. Aside from knowledge of these resources and tools, a common hindrance is understanding the ease of use and estimating time and cost. With respect to time, it is difficult to directly compare cloud-hosted environments to local-hardware given the diversity and dynamic nature of computational time and costs, and the fact that processing time is affected by everything from algorithm, general code and settings optimization, to the processing pipeline's physical architecture used for the cloud-hosted environment. For this reason, an exploratory analysis such as presented here can help estimate the scale of time and costs. Since different search algorithms will use resources differently and react differently to search settings (e.g., mass tolerance, database size, variable modifications, and quantification), users should benchmark their preferred tools with their typical datasets. Broader efforts such as the ongoing proteomic data analysis pipeline comparison led by the ELIXR Proteomics Community ${ }^{41}$ will help clarify pipeline performance. For the discussion herein, a previously published study ${ }^{42}$ with a follow-up analysis ${ }^{43}$ of label-free data-dependent acquisition shotgun proteomic data from mammalian urine was chosen. For this example, the data was analyzed using AWS Elastic Compute Cloud (EC2) instances with an arbitrarily chosen OpenMS-based Comet-Percolator workflow constructed with KNIME (Figure 2). The 
goal was to demonstrate time and costs with different computational resources on a ubiquitous commercial platform using a typical label-free data-dependent shotgun proteomic experiment.

\section{Example Cloud Set-up}

Although AWS EC2 was chosen for this example, most services offer similar remote desktop access. This means that even for the cloud novice, making productive use of instances does not require command line work, but instead can look just like the computer they are already using by access via remote clients such as Windows Remote Desktop (RDP), Virtual Network Computing (VNC), Team Viewer or other available remote desktop options. For this example, a community Amazon Machine Image (AMI: Windows_Server-2019-English-Full-Base2020.09.09) was used as a c5d.xlarge instance to set up the software and run analysis. The c5d instance types provide very fast local (to the instance's motherboard) scratch disk space that is erased upon shut down of the on-demand instance. The 20 raw files from PRIDE PXD00901944 of approximately 1.3 gigabytes each were copied to the instance using FTP. It should be noted that transfer speeds tend to be faster between a public cloud-hosted environment and a public data repository since they tend to be network proximal; for example, there seem to be fewer hops and bigger pipes between MassIVE and XSEDE. Additional tools such as Globus or Aspera can make high transfer speed through proximity for data that are not in public repositories. Once the raw files were loaded onto the instance, MSConvert 3.0.20280 was installed to derive MS2 mzML files. The remove_duplicates.py script ${ }^{45}$ was used to collapse duplicate fasta entries prior to workflow execution. The KNIME 4.2.2 scientific workflow platform was installed with OpenMS 2.6.0 nodes and a simple workflow was built using KNIME OpenMS tutorials as a guide ${ }^{38,46}$. Broadly, a mix of OpenMS native nodes including DecoyDatabase, Peptidelndexer, PSMFeatureExtractor and IDMerger, and adapter nodes for the Comet search engine ${ }^{47}$ and Percolator ${ }^{48}$ were used, and the specific KNIME workflow with required files and settings for replication locally or in cloud-hosted environments is publicly available ${ }^{49}$. These different software were chosen to demonstrate the capabilities and possibilities of this approach. Following completion of the workflow with the c5d.xlarge instance type, outputs and benchmark times were saved from the scratch drive to a long-term EBS (Elastic Block Store) volume and the instance was shut down. Two further iterations were completed in the same manner by rebooting and re-running the same workflow using the c5d.2xlarge and c5d.12xlarge instance types. Only the threads parameter was changed and the resulting benchmarking information was saved after confirming the idXML outputs. For all three instance types tested, the number of parallel threads allowed to be used by the CometAdapter was set at one less than the number of cores available to the instance type. A representative completed search result was retained in long-term storage and, the results could be transferred elsewhere by various manners including browser-based file upload from the desktop environment before shutting down. 


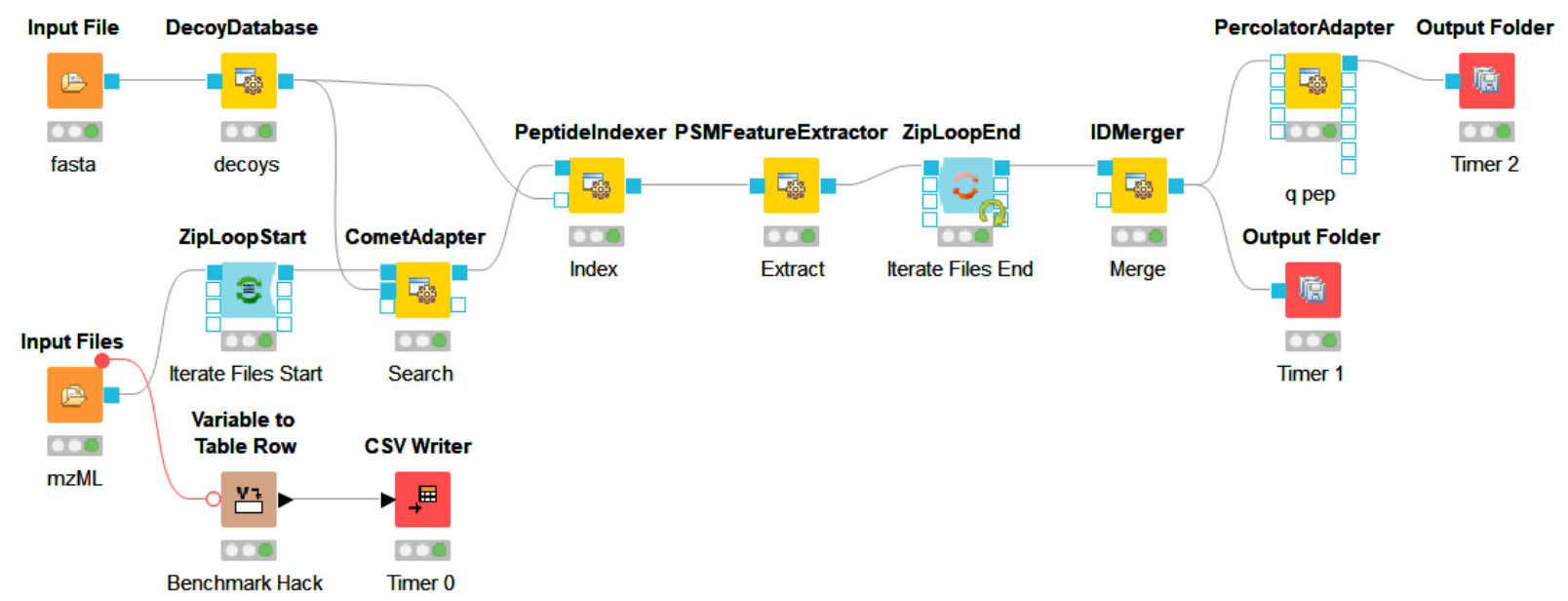

Figure 2. KNIME workflow using OpenMS adapters. OpenMS 2.6 KNIME nodes for a simple workflow loading spectra and protein sequence files, performing a search, and consolidating peptide and protein IDs found in the input spectra files. A simple approach to benchmarking was achieved through a standard KNIME node and file write timestamps.

\section{Time and Cost}

In the case of a commercial provider, it is recommended to use a modest computational tier with low hourly cost for learning the system and setting up the workflow. It is also important to note that on-demand pricing of services like EC2 requires instances to be manually shut down when not in use. For high-end instances, if not shut down, the monthly bill can easily exceed many thousand dollars. For this specific example, three c5d instance types were compared and analysis time and cost were determined using the same data, workflow and search parameters (Table 1). As stated before, the time and cost aspect of this comparison is extremely dynamic and will change depending on computational speeds of instances (which are periodically upgraded), software (and their updates) and search settings (e.g., number of variable modifications). This is also important to note when comparing to running on local resources or in other cloud-hosted environments. For comparison, there are benchmarks available for this specific data set analyzed on different hardware with different software, which can be found here ${ }^{50}$. Specific to the results described herein, unsurprisingly the search was quicker with the higher performance tiers, but this came at a monetary cost. Though this tradeoff is important to note, in situations where accessibility is the main concern, it demonstrates that a modestly powered instance can perform well at a low cost, especially since "set it and forget it" is a common approach when analyzing proteomic results locally. Still, any costs may prove prohibitive when resources are limited, though with federally subsidized resources like Jetstream, this is surmountable. Budgeting dynamic costs versus one-time hardware purchases is also difficult, but it is expected that this cost model will continue to be easier to cover as institutional views shift to preferring cloud-hosted environments versus local infrastructure. There are additional concerns beyond this discussion concerning privacy and security concerns of using cloud-hosted environments for certain types of data, and this may also affect costs. Whether speed or cost is a priority is up to each user and situation, but given the elastic nature 
of resource allocation, this decision can be made dynamically, further emphasizing the power of working in a cloud-hosted environment.

Table 1. Time and cost of running the same 20 injection search-only workflow on three instance types. The comparison of the large versus the $12 x$ large c5 instance found that the higher performance is about three times more expensive but six times faster.

\begin{tabular}{r|ccc}
\multicolumn{1}{c}{} & c5d.large & c5d.2xlarge & c5d.12xlarge \\
\cline { 2 - 4 } processors available & 2 & 8 & 48 \\
processors used & 1 & 7 & 47 \\
run rate $(\mathbf{\$} / \mathbf{h})$ & 0.22 & 0.84 & 5.08 \\
search time (hours:minutes) & $3: 54$ & $1: 15$ & $0: 31$ \\
total workflow time (hours:minutes) & $4: 01$ & $1: 21$ & $0: 37$ \\
cost (\$) & 0.88 & 1.13 & 3.13
\end{tabular}

\section{Result Handling and Reproducibility}

Best practices for using a cloud-hosted environment will vary across fields, but in proteomic data analysis the primary computational bottlenecks are file conversion, processing spectra, peptide identification, protein inference, and relative quantification if applicable. Downstream steps such as differential analysis or enrichment analysis can be performed with fewer computational resources, meaning these steps are likely more appropriate on local systems. Following completion of the search steps, results files can be retrieved to local workstations. Typically, result files can be explored using the same software used to generate the data or software-specific viewers (e.g., PeptideShaker for SearchGUI output). Alternatively, flat file exports (e.g., csv) may be shared between users. It is also possible to stay completely within the cloud by using one of a growing number of cloud-based services for statistical analysis and result sharing (e.g., SimpliFi $\left.{ }^{51}\right)$.

One of the most important benefits of using cloud-hosted environments is the opportunities for reproducibility ${ }^{52}$. Images can be shared privately or publicly between users, allowing others to reproduce the same operating system and software versions and, if desired, settings as the original analysis. To illustrate this point, the AMI used in this example has been shared publicly on EC2 (ami-0dead6b478bd16281 on us-east-2 region), and is ready for use following modification of KNIME memory allocation and CometAdapter threads to match the instance's resources. An image may also be cloned and modified if a user wants to update or change software, thus allowing for comparison of results all while preserving the original environment. Another way to achieve reproducibility is by using containers. For example, software from GitHub can be packaged into a Singularity container ${ }^{53}$ that can be linked with other workflow steps using Nextflow. In this way, more complex sets of software with different dependencies can work together in a pipeline that can be used in a cloud-hosted environment. The reason this is preferred is that software is exactly preserved and shared via GitHub and the container can also be made available via repositories such as Singularity Container Registry ${ }^{54}$, DockerHub $^{55}$ or BioContainers ${ }^{56}$, while optimized application-specific Nextflow workflows are 
available via nf-core ${ }^{57}$. Together, this degree of portability and reproducibility enables replication by anyone on any system.

\section{Real and Perceived Limitations}

With the concurrent advancements in proteomic data repositories and software along with pricing and performance of cloud-hosted environments, there are fewer limitations than ever to take proteomics to the cloud. In addition to the points addressed in previous sections, data transfer speed and storage costs present different limitations. Transferring data from repositories or from a private resource to cloud-hosted environments can be very fast depending on where the actual servers are located. Although it can be tedious and unintuitive, it is worthwhile to choose services and host locations with data sources in mind. In the future, there will be improved integration of data repositories with cloud-hosted resources (e.g., Google's Cloud Life Sciences public datasets), which will increase usability and reduce data storage costs. Currently, the cost of storage used with a computational instance will vary from free to minimal depending on the service, but it is a fraction of the computational cost if managed properly. Finally, maybe the most crucial limitation to adoption of many of the resources and tools is the perceived difficulty. The proteomics community could address this by creating more pre-built proteomic-centric images, while software and pipeline developers could provide detailed vignettes using real data on different cloud-hosted environments.

\section{Future Outlook}

When researchers look to the cloud it is often to accomplish tasks that are not possible with local workstations. Although proteomics researchers are adept at using local resources to accomplish large computational tasks, there is far-reaching potential in developing and utilizing cloud-hosted environments for proteomic needs. The resilience of using remote resources should not be understated in the current climate, and though not foolproof, they are lockdown proof. More importantly, as the global flow of people has slowed, utilizing the cloud to avoid lost time with trainees in other countries or to maintain research with distant colleagues is invaluable and can be facilitated using shared cloud resources. Moreover, these benefits are applicable beyond mass spectrometry-based proteomics since other similar mass spectrometry-based domains can benefit from cloud-hosted environments, including imaging, lipidomics, and metabolomics. As it becomes more common to work in cloud-hosted environments we will see benefits that will continue to drive the field forward.

\section{Acknowledgements}

The author wishes to thank Devin Drew (Thermo Fisher Scientific) for his assistance in implementing searches on AWS and for his expertise in using proteomic software in cloud environments. The author also wishes to thank Phillip Wilmarth (Oregon Health \& Science University) and Brian Beck (University of Texas at Austin) for critical insight and feedback. The identification of certain commercial equipment, instruments, software, or materials does not imply recommendation or endorsement by the National Institute of Standards and Technology, nor does it imply that the products identified are necessarily the best available for the purpose. 
Competing Interests: The authors declare that they have no competing financial interests.

Corresponding author: BN; benjamin.neely@nist.gov

Contributions: B.N. conceived and wrote the manuscript

\section{Glossary}

Cloud Computing: computing performed with remote resources. Arguably, the first "cloud" drawn as an information system visual was for the first transatlantic demonstration of connectivity among ARPANET, SATNET and PRNET in $1977 .{ }^{2}$

Container: an encapsulated environment typically containing only one program and associated dependencies, not to be confused with a virtual appliance, which is more akin to an image. A container can be run with a set of input and output arguments via a command or in a workflow.

Image: a complete snapshot ("template") of a computational environment including operating system.

Instance: the instantiation of an instance type (putting a machine image onto an instance type and 'spinning it up' in a server farm somewhere).

Instance type: a set of attributes describing number of processor cores, memory and I/O resources having a cost per unit time.

Pipeline: a generic term referring to linked steps of analysis. Workflow engines generate workflows, often referred to as pipelines.

Running instance: An instance that is accumulating cost attributable to its instance type and associated data storage and $\mathrm{I} / \mathrm{O}$.

Stopped (or not running) instance: An instance accumulating cost attributable only to the storage of its image.

Time-sharing (computing term): a concept dating from the 1950's relating to a computer system handling a number of problems (for different users) concurrently. ${ }^{1}$

Workflow engines: typically a visual tool to link steps that are wrappers or containers of other programs. May be used in different environments including clustering if capable. 


\section{References}

1. Lebo, H., 100 Days: How Four Events in 1969 Shaped America. Illustrated ed.; Rowman and Littlefield Publishers: 2019.

2. $\quad$ Cerf, V. G., The day the Internet age began. Nature 2009, 461 (7268), 1202-3.

3. Devisetty, U. K.; Tuteja, R. MAKER 2.31.9 with CCTOOLS Jetstream Tutorial.

https://cyverse.atlassian.net/wiki/spaces/TUT/pages/258736333/MAKER+2.31.9+with+CCTOO LS+Jetstream+Tutorial (accessed 26 October 2020).

$4 . \quad$ Running Cactus on AWS.

https://github.com/ComparativeGenomicsToolkit/cactus/blob/master/doc/running-in-aws.md (accessed 26 October 2020).

5. Bioconductor in the cloud. https://www.bioconductor.org/help/bioconductor-cloud-ami/ (accessed 16 November 2020).

6. Pino, L. K.; Rose, J.; O'Broin, A.; Shah, S.; Schilling, B., Emerging mass spectrometrybased proteomics methodologies for novel biomedical applications. Biochemical Society Transactions 2020, 48 (5), 1953-1966.

7. Heck, M.; Neely, B. A., Proteomics in Non-model Organisms: A New Analytical Frontier. Journal of proteome research 2020, 19 (9), 3595-3606.

8. $\quad$ Tsiamis, V.; lenasescu, H. I.; Gabrielaitis, D.; Palmblad, M.; Schwämmle, V.; Ison, J., One Thousand and One Software for Proteomics: Tales of the Toolmakers of Science. Journal of proteome research 2019, 18 (10), 3580-3585.

9. $\quad$ Eng, J. K.; McCormack, A. L.; Yates, J. R., An approach to correlate tandem mass spectral data of peptides with amino acid sequences in a protein database. Journal of the American Society for Mass Spectrometry 1994, 5 (11), 976-89.

10. Prakash, A.; Ahmad, S.; Majumder, S.; Jenkins, C.; Orsburn, B., Bolt: a New Age Peptide Search Engine for Comprehensive MS/MS Sequencing Through Vast Protein Databases in Minutes. Journal of the American Society for Mass Spectrometry 2019, 30 (11), 2408-2418.

11. ionbot. https://ionbot.cloud (accessed 26 October 2020).

12. Stewart, P. A.; Kuenzi, B. M.; Mehta, S.; Kumar, P.; Johnson, J. E.; Jagtap, P.; Griffin, T. J.; Haura, E. B., The Galaxy Platform for Reproducible Affinity Proteomic Mass Spectrometry Data Analysis. Methods in molecular biology (Clifton, N.J.) 2019, 1977, 249-261.

13. GalaxyP - Access to software. http://galaxyp.org/access-galaxy-p/ (accessed 26 October 2020).

14. Park, C. Y.; Klammer, A. A.; Käll, L.; MacCoss, M. J.; Noble, W. S., Rapid and accurate peptide identification from tandem mass spectra. Journal of proteome research 2008, 7 (7), 3022-7.

15. Searle, B. C.; Pino, L. K.; Egertson, J. D.; Ting, Y. S.; Lawrence, R. T.; MacLean, B. X.; Villén, J.; MacCoss, M. J., Chromatogram libraries improve peptide detection and quantification by data independent acquisition mass spectrometry. Nature communications 2018, $9(1), 5128$.

16. Kong, A. T.; Leprevost, F. V.; Avtonomov, D. M.; Mellacheruvu, D.; Nesvizhskii, A. I., MSFragger: ultrafast and comprehensive peptide identification in mass spectrometry-based proteomics. Nature methods 2017, 14 (5), 513-520.

17. FragPipe. https://github.com/Nesvilab/FragPipe (accessed 26 October 2020).

18. Chambers, M. C.; Maclean, B.; Burke, R.; Amodei, D.; Ruderman, D. L.; Neumann, S.; Gatto, L.; Fischer, B.; Pratt, B.; Egertson, J.; Hoff, K.; Kessner, D.; Tasman, N.; Shulman, N.; Frewen, B.; Baker, T. A.; Brusniak, M. Y.; Paulse, C.; Creasy, D.; Flashner, L.; Kani, K.; Moulding, C.; Seymour, S. L.; Nuwaysir, L. M.; Lefebvre, B.; Kuhlmann, F.; Roark, 
J.; Rainer, P.; Detlev, S.; Hemenway, T.; Huhmer, A.; Langridge, J.; Connolly, B.; Chadick, T.; Holly, K.; Eckels, J.; Deutsch, E. W.; Moritz, R. L.; Katz, J. E.; Agus, D. B.; MacCoss, M.; Tabb, D. L.; Mallick, P., A cross-platform toolkit for mass spectrometry and proteomics. Nature biotechnology 2012, 30 (10), 918-20.

19. Vaudel, M.; Barsnes, H.; Berven, F. S.; Sickmann, A.; Martens, L., SearchGUI: An open-source graphical user interface for simultaneous OMSSA and X!Tandem searches.

Proteomics 2011, 11 (5), 996-9.

20. Kohlbacher, O.; Reinert, K.; Gröpl, C.; Lange, E.; Pfeifer, N.; Schulz-Trieglaff, O.; Sturm, M., TOPP--the OpenMS proteomics pipeline. Bioinformatics (Oxford, England) 2007, 23 (2), e191-7.

21. Röst, H. L.; Sachsenberg, T.; Aiche, S.; Bielow, C.; Weisser, H.; Aicheler, F.; Andreotti, S.; Ehrlich, H. C.; Gutenbrunner, P.; Kenar, E.; Liang, X.; Nahnsen, S.; Nilse, L.; Pfeuffer, J.; Rosenberger, G.; Rurik, M.; Schmitt, U.; Veit, J.; Walzer, M.; Wojnar, D.; Wolski, W. E.; Schilling, O.; Choudhary, J. S.; Malmström, L.; Aebersold, R.; Reinert, K.; Kohlbacher, O., OpenMS: a flexible open-source software platform for mass spectrometry data analysis. Nature methods 2016, 13 (9), 741-8.

22. Keller, A.; Eng, J.; Zhang, N.; Li, X. J.; Aebersold, R., A uniform proteomics MS/MS analysis platform utilizing open XML file formats. Molecular systems biology 2005, 1, 2005.0017.

23. Deutsch, E. W.; Mendoza, L.; Shteynberg, D.; Farrah, T.; Lam, H.; Tasman, N.; Sun, Z.; Nilsson, E.; Pratt, B.; Prazen, B.; Eng, J. K.; Martin, D. B.; Nesvizhskii, A. I.; Aebersold, R., A guided tour of the Trans-Proteomic Pipeline. Proteomics 2010, 10 (6), 1150-9.

24. Fenyö, D.; Beavis, R. C., A method for assessing the statistical significance of mass spectrometry-based protein identifications using general scoring schemes. Analytical chemistry 2003, 75 (4), 768-74.

25. Cox, J.; Mann, M., MaxQuant enables high peptide identification rates, individualized p.p.b.-range mass accuracies and proteome-wide protein quantification. Nature biotechnology 2008, 26 (12), 1367-72.

26. Sinitcyn, P.; Tiwary, S.; Rudolph, J.; Gutenbrunner, P.; Wichmann, C.; Yılmaz, Ş.; Hamzeiy, H.; Salinas, F.; Cox, J., MaxQuant goes Linux. Nature methods 2018, 15 (6), 401. 27. Solntsev, S. K.; Shortreed, M. R.; Frey, B. L.; Smith, L. M., Enhanced Global Posttranslational Modification Discovery with MetaMorpheus. Journal of proteome research 2018, 17 (5), 1844-1851.

28. Cesnik, A. J.; Miller, R. M.; Ibrahim, K.; Lu, L.; Millikin, R. J.; Shortreed, M. R.; Frey, B. L.; Smith, L. M., Spritz: A Proteogenomic Database Engine. Journal of proteome research 2020.

29. Berthold, M. R.; Cebron, N.; Dill, F.; Gabriel, T. R.; Kötter, T.; Meinl, T.; Ohl, P.; Thiel, K.; Wiswedel, B., KNIME - the Konstanz information miner: version 2.0 and beyond. SIGKDD Explor. Newsl. 2009, 11 (1), 26-31.

30. Albrecht, M.; Donnelly, P.; Bui, P.; Thain, D. In Makeflow: A portable abstraction for data intensive computing on clusters, clouds, and grids, Proceedings of the 1st ACM SIGMOD Workshop on Scalable Workflow Execution Engines and Technologies, 2012; pp 1-13.

31. Di Tommaso, P.; Chatzou, M.; Floden, E. W.; Barja, P. P.; Palumbo, E.; Notredame, C., Nextflow enables reproducible computational workflows. Nature biotechnology 2017, 35 (4), 316-319.

32. Köster, J.; Rahmann, S., Snakemake--a scalable bioinformatics workflow engine.

Bioinformatics (Oxford, England) 2012, 28 (19), 2520-2.

33. Wilde, M.; Hategan, M.; Wozniak, J. M.; Clifford, B.; Katz, D. S.; Foster, I., Swift: A language for distributed parallel scripting. Parallel Computing 2011, 37 (9), 633-652.

34. Vivian, J.; Rao, A. A.; Nothaft, F. A.; Ketchum, C.; Armstrong, J.; Novak, A.; Pfeil, J.; Narkizian, J.; Deran, A. D.; Musselman-Brown, A.; Schmidt, H.; Amstutz, P.; Craft, B.; 
Goldman, M.; Rosenbloom, K.; Cline, M.; O'Connor, B.; Hanna, M.; Birger, C.; Kent, W. J.; Patterson, D. A.; Joseph, A. D.; Zhu, J.; Zaranek, S.; Getz, G.; Haussler, D.; Paten, B., Toil enables reproducible, open source, big biomedical data analyses. Nature biotechnology 2017, 35 (4), 314-316.

35. Mohammed, Y.; Mostovenko, E.; Henneman, A. A.; Marissen, R. J.; Deelder, A. M.; Palmblad, M., Cloud parallel processing of tandem mass spectrometry based proteomics data. Journal of proteome research 2012, 11 (10), 5101-8.

36. Sadygov, R. G.; Eng, J.; Durr, E.; Saraf, A.; McDonald, H.; MacCoss, M. J.; Yates, J. R., 3rd, Code developments to improve the efficiency of automated MS/MS spectra interpretation. Journal of proteome research 2002, 1 (3), 211-5.

37. UltraQuant. https://github.com/kentsisresearchgroup/UltraQuant (accessed 26 October 2020).

38. OpenMS Nodes for KNIME. https://www.knime.com/community/bioinf/openms (accessed 26 October 2020).

39. Perez-Riverol, Y.; Heumos, L.; Gabernet, G.; Garci, M., nf-core/proteomicslfq: v1.0.0 Lovely Logan. Zenodo 2020.

40. $\quad$ Chard, R.; Skluzacek, T. J.; Li, Z.; Babuji, Y.; Woodard, A.; Blaiszik, B.; Tuecke, S.; Foster, I.; Chard, K. J. a. p. a., Serverless supercomputing: High performance function as a service for science. arXiv 2019, arXiv:1908.04907.

41. Comparison, benchmarking and dissemination of proteomics data analysis pipelines. https://elixir-europe.org/about-us/commissioned-services/proteomics-pipelines (accessed 26 October 2020).

42. Neely, B. A.; Prager, K. C.; Bland, A. M.; Fontaine, C.; Gulland, F. M.; Janech, M. G., Proteomic Analysis of Urine from California Sea Lions ( Zalophus californianus): A Resource for Urinary Biomarker Discovery. Journal of proteome research 2018, 17 (9), 3281-3291.

43. Wilmarth, P. Sea lion urine SpC. https://github.com/pwilmart/Sea lion urine SpC (accessed 26 October 2020).

44. PRIDE Project PXD009019.

ftp://ftp.pride.ebi.ac.uk/pride/data/archive/2018/06/PXD009019 (accessed 24 September 2020).

45. fasta_utilities. https://github.com/pwilmart/fasta utilities/ (accessed 26 October 2020).

46. OpenMS Tutorial. https://sourceforge.net/p/open-

$\mathrm{ms} / \mathrm{code} / \mathrm{HEAD} / \mathrm{tree} / T u t o r i a l s / U M$ 2014/Handout/handout.pdf?format=raw (accessed 26

October 2020).

47. Eng, J. K.; Jahan, T. A.; Hoopmann, M. R., Comet: an open-source MS/MS sequence database search tool. Proteomics 2013, 13 (1), 22-4.

48. The, M.; MacCoss, M. J.; Noble, W. S.; Käll, L., Fast and Accurate Protein False Discovery Rates on Large-Scale Proteomics Data Sets with Percolator 3.0. Journal of the American Society for Mass Spectrometry 2016, 27 (11), 1719-1727.

49. Neely, B. A., An example KNIME-OpenMS workflow for benchmarking cloud-hosted environments. Zenodo 2020.

50. Cloud_desktop_benchmarks. https://github.com/pwilmart/Cloud desktop benchmarks (accessed 26 October 2020 ).

51. SimpliFi. https://simplifi.protifi.com/ (accessed 26 October 2020).

52. Madduri, R.; Chard, K.; D'Arcy, M.; Jung, S. C.; Rodriguez, A.; Sulakhe, D.; Deutsch, E.; Funk, C.; Heavner, B.; Richards, M.; Shannon, P.; Glusman, G.; Price, N.; Kesselman, C.; Foster, I., Reproducible big data science: A case study in continuous FAIRness. PloS one 2019, 14 (4), e0213013.

53. The Singularity Recipe. https://singularity.lbl.gov/docs-recipes (accessed 26 October 2020).

54. Singularity Container Registry. https://singularity-hub.org/ (accessed 26 October 2020). 55. Docker Hub. https://hub.docker.com/ (accessed 26 October 2020). 
56. Bai, J.; Bandla, C.; Guo, J.; Alvarez, R. V.; Vizcaíno, J. A.; Bai, M.; Moreno, P.; Grüning, B. A.; Sallou, O.; Perez-Riverol, Y., BioContainers Registry: searching for bioinformatics tools, packages and containers. 2020, 2020.07.21.187609.

57. Ewels, P. A.; Peltzer, A.; Fillinger, S.; Patel, H.; Alneberg, J.; Wilm, A.; Garcia, M. U.; Di Tommaso, P.; Nahnsen, S., The nf-core framework for community-curated bioinformatics pipelines. Nature biotechnology 2020, 38 (3), 276-278. 